\title{
Math-Net.Ru
}

Общероссийский математический портал

П. С. Геворкян, Об одном критерии подвижности, Матем. заметки, 2002, том 71, выпуск 2, 311-315

DOI: https://doi.org/10.4213/mzm642 
Использование Общероссийского математического портала Math-Net.Ru подразумевает, что вы прочитали и согласны с пользовательским соглашением http://www. mathnet.ru/rus/agreement

Параметры загрузки:

IP: 34.239 .49 .27

26 апреля 2023 г., 18:07:19

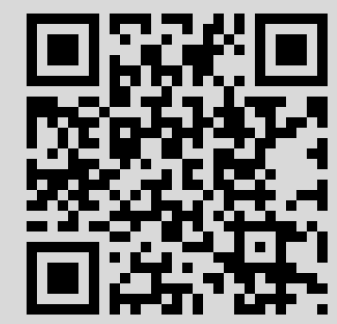




\section{ОБ ОДНОМ КРИТЕРИИ ПОДВИЖНОСТИ}

\section{П. С. Геворкян}

Понятие подвижности первоначально, для метризуемых компактов, было введено Борсуком [1]. На более общие случаи было перенесено Богатым [2], Мардешичем и Сегалом [3], Сегалом [4].

Следует отметить, что подвижность топологического пространства в указанных работах была определена либо с помощью окрестностей данного пространства (замкнуто вложенного в некотоpoе $A R$-пространство), либо с помощью обратных спектров, в зависимости от того, какой подход к теории шейпов применяется. Однако при категорном подходе Мардешича [5] к теории шейпов не было дано подходящее категорное определение подвижности.

В настоящей статье к понятию подвижности топологического пространства применяется новьй категорный подход. Вводится понятие подвижной категории и доказьвается, что подвижность топологического пространства равносильна подвижности некоторой, соответствующей ему категории, определенной Мардешичем [5].

Пусть $K$-произвольная категория, а $K^{\prime}$ - произвольная ее подкатегория.

ОПРЕДЕЛЕниЕ 1. Скажем, что подкатегория $K^{\prime}$ подвижна в категории $K$, если для произвольного объекта $X \in \mathrm{ob}\left(K^{\prime}\right)$ существуют объект $Y \in \mathrm{ob}\left(K^{\prime}\right)$ и морфизм $f \in \operatorname{Mor}_{K^{\prime}}(Y, X)$ такие, что для любого объекта $Z \in \mathrm{ob}\left(K^{\prime}\right)$ и любого морфизма $g \in \operatorname{Mor}_{K^{\prime}}(Z, X)$ существует морфизм $h \in \operatorname{Mor}_{K}(Y, Z)$ такой, что коммутативна диаграмма 1.

ОПРЕДЕЛЕНИЕ 2. Категорию $K$ назовем подви жной, если она подвижна относительно самой себя.

(C) П.С. ГЕворкян

2002 


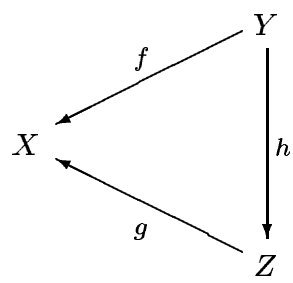

\section{ДиАГРАМма 1}

ОПРЕДЕЛЕНИЕ 3 [6]. Говорят, что $K$ есть категория с нулевыми морфизмами, если для любой пары $(A, B)$ объектов из категории $K$ существуют морфизмы $o_{B} A: A \rightarrow B$ такие, что для всех морфизмов $\nu: B \rightarrow C$ и $u: D \rightarrow A$, где $C$ и $D$ - произвольные объекты категории $K$, выполняется

$$
\nu_{B A}=o_{C A}, \quad o_{B A} u=o_{B D}
$$

ОПРЕДЕЛЕНИЕ 4 [6]. Объект $O \in \mathrm{ob}(K)$ назьвается инициальныл, если для любого объекта $X \in \mathrm{ob}(K)$ множество $\operatorname{Mor}_{K}(O, X)$ состоит из единственного морфизма.

ТЕОРема 1. Всякая категория $K$ с нулевыми морфизмами подвижна.

ДокАЗАтЕльство. Пусть $X \in \mathrm{ob}(K)$ - произвольньй объект. Оказывается, что в качестве искомого объекта (см. определение 1) можно взять произвольный объект $Y \in \mathrm{ob}(K)$, а в качестве морфизма $f \in K(Y, X)$ следует взять нулевой морфизм $o_{X Y}: Y \rightarrow X$. В самом деле, рассмотрим произвольньй морфизм $g \in K(Z, X)$. Теперь очевидно, что нулевой морфизм $o_{Z Y}: Y \rightarrow Z$ искомьй, т.е. имеет место равенство $g_{Z Y}=o_{X Y}$, которое следует из определения 3.

ТЕорема 2. Всякая категория $K$, имеющая инициальный обвект, подвижна.

ДокАЗАТЕльство. Пусть $X \in \mathrm{ob}(K)$ - произвольный объект. Рассмотрим инициальньй объект $O$ категории $K$. Единственный морфизм из объекта $O$ в объект $X$ обозначим через $u_{X}$. Теперь нетрудно заметить, что объект $O$ и морфизм $u_{X}: O \rightarrow X$ являются искомыми. В самом деле, пусть $Y \in \mathrm{ob}(K)$ - произвольный объект, а $g: Y \rightarrow X-$ произвольньй морфизм. Очевидно, что единственный морфизм $u_{Y}: O \rightarrow Y$ удовлетворяет условию $u_{X}=g \circ u_{Y}$.

При категорном подходе к теории шейпов [5] каждому топологическому пространству $X$ сопоставляется некоторая категория $W^{X}$, объекты которой являются гомотопическими классами $f: X \rightarrow Q$, а морфизмы - коммутативньми треугольниками диаграммы 2, где $Q, Q^{\prime}-A N R$-пространства.

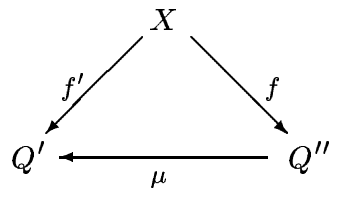

\section{ДИАГРАМмА 2}

Теорема 3. Топологическое пространство X подвижно тогда и только тогда, когда подвихна категория $W^{X}$.

Последняя теорема есть простая переформулировка следующей теоремы.

Теорема 4. Топологическое пространство $X$ подвижно тогда и только тогда, когда выполняется следующее условие:

(*) для произвольного ANR-пространства $Q$ илюбого гомотопического класса $f: X \rightarrow Q$ существуют ANR-пространство $Q^{\prime}$, гомотопические классы $f^{\prime}: X \rightarrow Q^{\prime}$ и $\eta: Q^{\prime} \rightarrow Q$ с условием $f=\eta \circ f^{\prime}$ такие, что, каковы бы ни были ANR-пространство $Q^{\prime \prime}$, гомотопические классы $f^{\prime \prime}: X \rightarrow Q^{\prime \prime}$ u $\eta^{\prime}: Q^{\prime \prime} \rightarrow Q$ с условием $f=\eta^{\prime} \circ f^{\prime \prime}$, существует гомотопический класс $\eta^{\prime \prime}: Q^{\prime} \rightarrow Q^{\prime \prime}$ такой, что выполняется равенство $\eta=\eta^{\prime} \circ \eta^{\prime \prime}$ (диаграмма 3). 


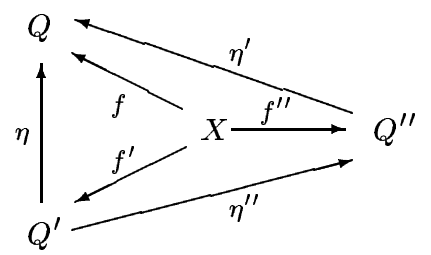

\section{ДиАГРАМма 3}

ДокАЗАТЕЛЬСтво. Пусть выполняется условие (*). Докажем, что пространство $X$ подвижно. Для этого рассмотрим некоторьй ассоциированный с $X$ спектр $\left\{X_{\alpha}, p_{\alpha \alpha^{\prime}}, A\right\}$ (см. [7]) и докажем, что он подвижен. Пусть $\alpha \in A$-произвольньй элемент, а $p_{\alpha}: X \rightarrow X_{\alpha}$-естественная проекция (диаграмма 4). Для отображения $p_{\alpha}: X \rightarrow X_{\alpha}$ рассмотрим подходящие $A N R$-пространство $Q^{\prime}$, гомотопические классы $f^{\prime}: X \rightarrow Q^{\prime}$ и $\eta: Q^{\prime} \rightarrow X_{\alpha}$ с условием $\eta \circ f^{\prime}=p_{\alpha}$, существование которых следует из условия $(*)$.

Так как ass $X=\left\{X_{\alpha}, p_{\alpha \alpha^{\prime}}, A\right\}$, то для $f^{\prime}: X \rightarrow Q^{\prime}$ существуют $\widetilde{\alpha} \in A, \widetilde{\alpha} \geqslant \alpha$, и $\widetilde{f}^{\prime}: X_{\widetilde{\alpha}} \rightarrow Q^{\prime}$ такие, что

Нетрудно убедиться, что

$$
f^{\prime}=\tilde{f}^{\prime} \circ p_{\widetilde{\alpha}}
$$

$$
p_{\alpha \widetilde{\alpha}} \circ p_{\widetilde{\alpha}}=\eta \circ \widetilde{f}^{\prime} \circ p_{\tilde{\alpha}}
$$

В самом деле,

$$
\eta \circ \widetilde{f}^{\prime} \circ p_{\widetilde{\alpha}}=\eta \circ f^{\prime}=p_{\alpha}=p_{\alpha \widetilde{\alpha}} \circ p_{\widetilde{\alpha}} .
$$

В силу равенства (1) и из определения ассоциированности следует, что существует индекс $\alpha^{\prime} \in A$, $\alpha^{\prime} \geqslant \widetilde{\alpha}$, такой, что

$$
p_{\alpha \widetilde{\alpha}} \circ p_{\widetilde{\alpha} \alpha^{\prime}}=\eta \circ \widetilde{f}^{\prime} \circ p_{\widetilde{\alpha} \alpha^{\prime}}
$$

Оказьвается, что так найденный индекс $\alpha^{\prime} \in A$ удовлетворяет условию подвижности спектра $\left\{X_{\alpha}, p_{\alpha \alpha^{\prime}}, A\right\}$. В самом деле, пусть $\alpha^{\prime \prime} \in A, \alpha^{\prime \prime} \geqslant \alpha,-$ произвольный элемент. Для отображений $p_{\alpha \alpha^{\prime \prime}}: X_{\alpha^{\prime \prime}} \rightarrow X_{\alpha}$ и $p_{\alpha^{\prime \prime}}: X \rightarrow X_{\alpha^{\prime \prime}}$ (с условием $p_{\alpha}=p_{\alpha \alpha^{\prime \prime}} \circ p_{\alpha^{\prime \prime}}$ ) в силу условия $(*)$ существует отображение $\eta^{\prime \prime}: Q^{\prime} \rightarrow X_{\alpha^{\prime \prime}}$ такое, что

$$
\eta=p_{\alpha \alpha^{\prime \prime}} \circ \eta^{\prime \prime} \text {. }
$$

Теперь нетрудно убедиться, что отображение $g=\eta^{\prime \prime} \circ \widetilde{f}^{\prime} \circ p_{\widetilde{\alpha} \alpha^{\prime}}$ искомое, т.е. имеет место равенство

$$
p_{\alpha \alpha^{\prime}}=p_{\alpha \alpha^{\prime \prime}} \circ g \text {. }
$$

В самом деле,

$$
p_{\alpha \alpha^{\prime}}=p_{\alpha \widetilde{\alpha}} \circ p_{\widetilde{\alpha} \alpha^{\prime}}=\eta \circ \widetilde{f}^{\prime} \circ p_{\widetilde{\alpha} \alpha^{\prime}}=p_{\alpha \alpha^{\prime \prime}} \circ \eta^{\prime \prime} \circ \widetilde{f}^{\prime} \circ p_{\widetilde{\alpha} \alpha^{\prime}}=p_{\alpha \alpha^{\prime \prime}} \circ g .
$$

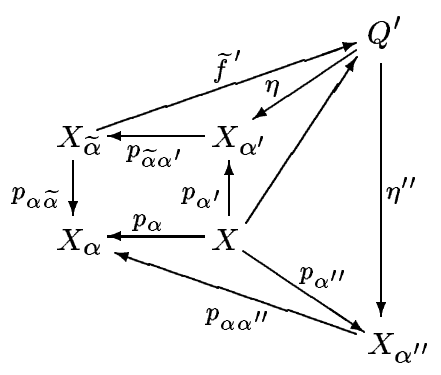


Теперь докажем обратное. Пусть $X$ - подвижное пространство, а $\left\{X_{\alpha}, p_{\alpha \alpha^{\prime}}, A\right\}$ - некоторый ассоцированный с ним обратный спектр. Докажем, что выполняется условие $(*)$.

Рассмотрим произвольный гомотопический класс $f: X \rightarrow Q$ (диаграмма 5). В силу ассоциированности спектра $\left\{X_{\alpha}, p_{\alpha \alpha^{\prime}}, A\right\}$ с пространством $X$ существует индекс $\alpha \in A$ и гомотопический класс $f_{\alpha}: X_{\alpha} \rightarrow Q$ такие, что

$$
f=f_{\alpha} \circ p_{\alpha} .
$$

Для найденного $\alpha \in A$ существует индекс $\alpha^{\prime} \in A, \alpha^{\prime} \geqslant \alpha$, который удовлетворяет условию подвижности спектра $\left\{X_{\alpha}, p_{\alpha \alpha^{\prime}}, A\right\}$. Из равенства (2) следует

$$
f=f_{\alpha} \circ p_{\alpha \alpha^{\prime}} \circ p_{\alpha^{\prime}}
$$

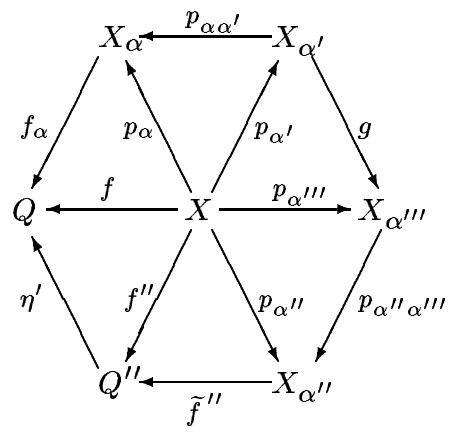

ДИАГРАМма 5

Теперь убедимся, что $A N R$-пространство $X_{\alpha^{\prime}}$, гомотопические классы $p_{\alpha^{\prime}}: X \rightarrow X_{\alpha^{\prime}}$ и $f_{\alpha}$ 。 $p_{\alpha \alpha^{\prime}}: X_{\alpha^{\prime}} \rightarrow Q$ удовлетворяют условию $(*)$. В самом деле, пусть $Q^{\prime \prime}$ - произвольное $A N R$-пространство, а $f^{\prime \prime}: X \rightarrow Q^{\prime \prime}$ и $\eta^{\prime}: Q^{\prime \prime} \rightarrow Q$ - такие гомотопические классы, которые удовлетворяют условию

$$
f=\eta^{\prime} \circ f^{\prime \prime} \text {. }
$$

Для гомотопического класса $f^{\prime \prime}: X \rightarrow Q^{\prime \prime}$ существуют индекс $\alpha^{\prime \prime} \in A, \alpha^{\prime \prime} \geqslant \alpha$, и гомотопический класс $\tilde{f}^{\prime \prime}: X_{\alpha^{\prime \prime}} \rightarrow Q^{\prime \prime}$ такие, что выполняется

$$
f^{\prime \prime}=\tilde{f}^{\prime \prime} \circ p_{\alpha^{\prime \prime}}
$$

Ясно, что

$$
f_{\alpha} \circ p_{\alpha \alpha^{\prime \prime}} \circ p_{\alpha^{\prime \prime}}=\eta^{\prime} \circ \tilde{f}^{\prime \prime} \circ p_{\alpha^{\prime \prime}} .
$$

Следовательно, согласно определению ассоциированности можно найти индекс $\alpha^{\prime \prime \prime} \in A, \alpha^{\prime \prime \prime} \geqslant \alpha^{\prime \prime}$, который удовлетворяет условию

$$
f_{\alpha} \circ p_{\alpha \alpha^{\prime \prime}} \circ p_{\alpha^{\prime \prime} \alpha^{\prime \prime \prime}}=\eta^{\prime} \circ \tilde{f}^{\prime \prime} \circ p_{\alpha^{\prime \prime} \alpha^{\prime \prime \prime}} .
$$

В силу подвижности спектра $\left\{X_{\alpha}, p_{\alpha \alpha^{\prime}}, A\right\}$ существует гомотопический класс $g: X_{\alpha^{\prime}} \rightarrow X_{\alpha^{\prime \prime \prime}}$ такой, что

$$
p_{\alpha \alpha^{\prime}}=p_{\alpha \alpha^{\prime \prime}} \circ p_{\alpha^{\prime \prime} \alpha^{\prime \prime \prime}} \circ g \text {. }
$$

Положим $\eta^{\prime \prime}=\widetilde{f}^{\prime \prime} \circ p_{\alpha^{\prime \prime}} \alpha^{\prime \prime \prime} \circ g$. Остается заметить, что гомотопический класс $\eta^{\prime \prime}: X_{\alpha^{\prime}} \rightarrow Q^{\prime \prime}$ удовлетворяет условию

$$
f_{\alpha} \circ p_{\alpha \alpha^{\prime}}=\eta^{\prime} \circ \eta^{\prime \prime}
$$

В самом деле,

$$
\eta^{\prime} \circ \eta^{\prime \prime}=\eta^{\prime} \circ \tilde{f}^{\prime \prime} \circ p_{\alpha^{\prime \prime} \alpha^{\prime \prime \prime}} \circ g=f_{\alpha} \circ p_{\alpha \alpha^{\prime \prime}} \circ p_{\alpha^{\prime \prime} \alpha^{\prime \prime \prime}} \circ g=f_{\alpha} \circ p_{\alpha \alpha^{\prime}} .
$$

Теорема доказана.

ЗАмечание 1 . Условие $(*)$ теоремы 4 можно принять за определение подвижности топологического пространства при категорном подходе к теории шейпов. 


\section{СПИСОК ЦИТИРОВАННОЙ ЛИТЕРАТУРЫ}

1. Borsuk K. // Fund. Math. 1969. V. 66. № 1. P. 137-146. 2. Богатый C. А. // Матем. сб. 1974. T. 93. №1. C. 90-102. 3. Mardesic S., Segal J. // Bull. Acad. Polon. Sci. 1970. V. 18. № 11. P. 649-654. 4. Segal J. // Lecture Notes in Math. 1974. № 375. P. 236-241. 5. Mardesic S., Segal J. Shape Theory - The Inverse System Approach. Amsterdam: North-Holland, 1982. 6. Бyкур И., Делиану А. Введение в теорию категорий и функторов. М., 1972. 7. Morita K. // Fund. Math. 1975. V. 86. P. 251-259.

Московский государственный универстет им. М.В.Ломоносова 